\title{
Fetal growth and insulin secretion in adult life
}

\author{
D. I. W. Phillips ${ }^{1}$, S. Hirst ${ }^{1}$, P.M.S.Clark ${ }^{2}$, C.N.Hales ${ }^{2}$, C.Osmond ${ }^{3}$ \\ ${ }^{1}$ Metabolic Programming Group, University of Southampton, Southampton General Hospital, Southampton, United Kingdom \\ ${ }^{2}$ Department of Clinical Biochemistry, University of Cambridge, Addenbrooke's Hospital, Cambridge, United Kingdom \\ ${ }^{3}$ MRC Environmental Epidemiology Unit, University of Southampton, Southampton General Hospital, Southampton, \\ United Kingdom
}

\begin{abstract}
Summary Recent studies suggest that NIDDM is linked with reduced fetal and infant growth. Observations on malnourished infants and studies of experimental animals exposed to protein energy or protein deficiency in fetal or early neonatal life suggest that the basis of this link could lie in the detrimental effects of poor early nutrition on the development of the beta cells of the islets of Langerhans. To test this hypothesis we have measured insulin secretion following an IVGTT in a sample of 82 normoglycaemic and 23 glucose intolerant subjects who were born in Preston, England, and whose birthweight and body size had been recorded at birth. The subjects with impaired glucose tolerance had lower first phase insulin secretion than the normoglycaemic
\end{abstract}

subjects (mean plasma insulin concentrations $3 \mathrm{~min}$ after intravenous glucose 416 vs $564 \mathrm{pmol} / \mathrm{l}, p=0.04$ ). Insulin secretion was higher in men than women (601 vs $457 \mathrm{pmol} / \mathrm{l}, p=0.02)$ and correlated with fasting insulin level $(p=0.04)$. However, there was no relationship between insulin secretion and the measurements of prenatal growth in either the normoglycaemic or glucose intolerant subjects. These results argue against a major role for defective insulin secretion as a cause of glucose intolerance in adults who were growth retarded in prenatal life. [Diabetologia (1994) 37:592-596]

Key words NIDDM, insulin secretion, fetal growth, programming.
Recent research has suggested that NIDDM may originate through impaired development in fetal life and early infancy. Among 370 men aged 64 years living in Hertfordshire, England, the percentage who had either NIDDM or impaired glucose tolerance was highest in those who had lowest birthweights or weights at age 1 year [1]. This finding was confirmed by a subsequent study of men and women aged 46 to 54 years who were born in Preston, England, where the association was shown to be due to babies who were small regardless of their gestational age rather than babies who were born

Received: 19 July 1993

and in revised form: 20 December 1993

Corresponding author: Dr. D. I. W. Phillips, Metabolic Programming Group, University of Southampton, Southampton General Hospital, Tremona Road, Southampton SO9 4XY, UK

Abbreviations: NIDDM, Non-insulin-dependent diabetes; OGTT, oral glucose tolerance test; IVGTT, intravenous glucose tolerance test. prematurely [2]. Associations between reduced fetal growth and glucose intolerance have also been described in Hispanic Americans and in the Pima Indians $[3,4]$.

Although impairment of insulin action and reduced insulin secretion are both important in the pathogenesis of NIDDM, it is not clear whether the association between early growth and diabetes is mediated by alterations in insulin resistance, defects in insulin secretion or a combination of both factors. A link between insulin resistance and fetal growth is suggested by the association between low birthweight and the development of syndrome $X$, the co-existence of impaired glucose intolerance, hypertension and hypertriglyceridaemia, and by a study of a subset of patients in the Preston study which showed that babies who were thin at birth were insulin resistant in adult life $[5,6]$. It is not known whether insulin secretion is also determined by early growth. There is, however, considerable evidence that poor nutrition in fetal and early infant life can be detrimental to the development of the beta cells of the 
islets of Langerhans and that this could lead to insulin deficiency in adult life. Much of the development of the islet cells occurs in utero [7]. Growth retarded newborn infants and malnourished children have reduced insulin responses to glucose together with reduced numbers of pancreatic beta cells [7, 8]. Animal studies also show that a period of protein energy malnutrition or even protein deficiency alone imposed in fetal or early postnatal life permanently impairs the insulin response to glucose $[9,10]$. Therefore to determine whether fetal growth is related to insulin secretion in adult life, we have measured insulin secretion following an IVGTT in a sample of the men and women who took part in the Preston study [2].

\section{Subjects and methods}

In Preston a standardised record form was kept for each woman admitted to the labour ward at Sharoe Green Hospital during 1935-1943. The record included the baby's birthweight, placental weight, length from crown to heel, and head circumference. Weights were measured in pounds ( 1 pound $=0.45 \mathrm{~kg}$ ) and lengths and head circumferences in inches $(1$ inch $=2.54 \mathrm{~cm})$ and were often rounded. The records also included the date of the mother's last menstrual period which we used to estimate the duration of gestation. As previously described [2] we used the $\mathrm{Na}$ tional Health Service Central Register to trace 393 of the singleton infants born in Sharoe Green Hospital, Preston during 19351943 who still live in or close to the city. After excluding six subjects previously diagnosed with diabetes, the remainder were asked to have an OGTT (based on the administration of 75-g of anhydrous glucose to subjects who had fasted overnight with 0 , $30 \mathrm{~min}$ and 2 -h sampling). Of the 266 subjects who had an OGTT, 232 were normoglycaemic ( 2 -h plasma glucose concentration below $7.8 \mathrm{mmol} / \mathrm{l}$ ), 29 had impaired glucose tolerance (2-hplasma glucose concentration of 7.8 to $11.1 \mathrm{mmol} / \mathrm{l})$ and 5 subjects had $\operatorname{NIDDM}$ (2-h plasma glucose concentration of over $11.1 \mathrm{mmol} / \mathrm{l}$ ).

At the clinic the subjects' height was measured with a portable stadiometer (CMS Weighing Equipment Ltd, Camden, London, UK). Weight was measured with a SECA scale (SECA Ltd, Birmingham, UK) and waist and hip circumferences with a steel tape measure. Subjects were also asked about their smoking and drinking habits. Alcohol consumption was converted into the total number of units each week ( 1 unit $=10$ g ethanol). Occupation of the father was used to define social class at birth, and current social class was derived from the subject's or husband's occupation.

Because NIDDM and impaired glucose tolerance were not only associated with low birthweight but also low birthweight in relation to placental weight, which is thought to be a marker of fetal malnutrition [2], we selected a sample from the normoglycaemic men and women in Preston whose birthweights and placental weights were spread over the range for these two variables. The sample was chosen from the three birthweight groups, $(\leq 2.9,-3.4$ and $>3.4 \mathrm{~kg}$ ) and the four placental weight groups $(\leq 0.45,-0.6,-0.7$ and $\geq 0.7 \mathrm{~kg}$ ) used in our previous analyses [2, 6]. Initially 4 men and 4 women were randomly selected from each of the 12 birthweight and placental weight groups, of whom 23 declined to take part. Where possible we replaced them with other people of the same sex from the same birthweight and placental weight groups. There were insufficient numbers in some groups and the final sample therefore comprised $82 \mathrm{sub}$ jects ( 42 men and 40 women). We also asked the men and
Table 1. Mean 3-min insulin secretion following intravenous glucose in men and women aged $47-56$ years according to glucose tolerance, sex, age, obesity and fasting insulin concentrations

\begin{tabular}{|c|c|c|}
\hline & $\begin{array}{l}\text { Mean 3-min } \\
\text { insulin secretion }\end{array}$ & $p$ value for trend \\
\hline $\begin{array}{l}\text { Glucose tolerance } \\
\text { Normoglycaemic } \\
\text { Impaired glucose } \\
\text { tolerance }\end{array}$ & $564(82)$ & 0.04 \\
\hline $\begin{array}{l}\text { Sex } \\
\text { Men } \\
\text { Women }\end{array}$ & $\begin{array}{ll}601 & (55) \\
457 & (50)\end{array}$ & 0.02 \\
\hline $\begin{array}{l}\text { Age (years) } \\
\leq 49 \\
-53 \\
>53\end{array}$ & $\begin{array}{l}555(31) \\
544(32) \\
496(42)\end{array}$ & 0.44 \\
\hline $\begin{array}{l}\text { Body mass index } \\
\leq 24 \\
-26 \\
-28 \\
>28\end{array}$ & $\begin{array}{l}533(35) \\
460(28) \\
529(17) \\
602(25)\end{array}$ & 0.38 \\
\hline $\begin{array}{l}\text { Waist to hip ratio } \\
\leq 0.82 \\
-0.89 \\
-0.94 \\
>0.94\end{array}$ & $\begin{array}{l}480(31) \\
526(25) \\
497(24) \\
628(25)\end{array}$ & 0.16 \\
\hline $\begin{array}{l}\text { Fasting insulin }(p r \\
\leq 30 \\
-45 \\
-60 \\
>60\end{array}$ & $\begin{array}{l}435(24) \\
607(27) \\
413(26) \\
681(28)\end{array}$ & 0.06 \\
\hline Total & $564(105)$ & \\
\hline
\end{tabular}

Numbers of subjects in parenthesis;

Geometric standard deviation of 3 -min plasma insulin $=1.86$

women found to have impaired glucose tolerance to undergo an IVGTT of whom 23 agreed. The IVGTTs were carried out between 08.30 and 11.00 hours. Subjects had fasted for 12 -h overnight and had been asked to abstain from alcohol and not to smoke during the fasting period. Dextrose in a $50 \%$ solution was administered into a large antecubital vein in a dose of $0.3 \mathrm{~g} / \mathrm{kg}$ body weight over 2 min by constant infusion. Arterialised venous blood was sampled from a retrogradely cannulated vein on the dorsum of the opposite hand $0,1,2,3,4,5,6,8$ and 10 min from the start of the dextrose infusion. We have previously shown the 3-min insulin concentration to be highly reproducible and this was therefore used as the measure of first phase insulin secretion [11]. To 'arterialise' the venous blood, the hand was placed in a water bath held at a constant temperature of $43^{\circ} \mathrm{C}$ for 10 min prior to the start of the infusion and kept there until the end of the study. Ethical approval for the study was obtained from the Preston District Hospital Ethics Committee and each participating subject gave informed consent.

\section{Assays and statistical methods}

Plasma glucose was measured by the hexokinase method and plasma insulin by two site immunometric assays with either ${ }^{125} \mathrm{I}$ or alkaline phosphatase as labels $[12,13]$. The interassay coefficient of variation of the insulin measurements was less than 
Table 2. Mean 3-min insulin secretion following intravenous glucose according to measurements of body size at birth and duration of gestation

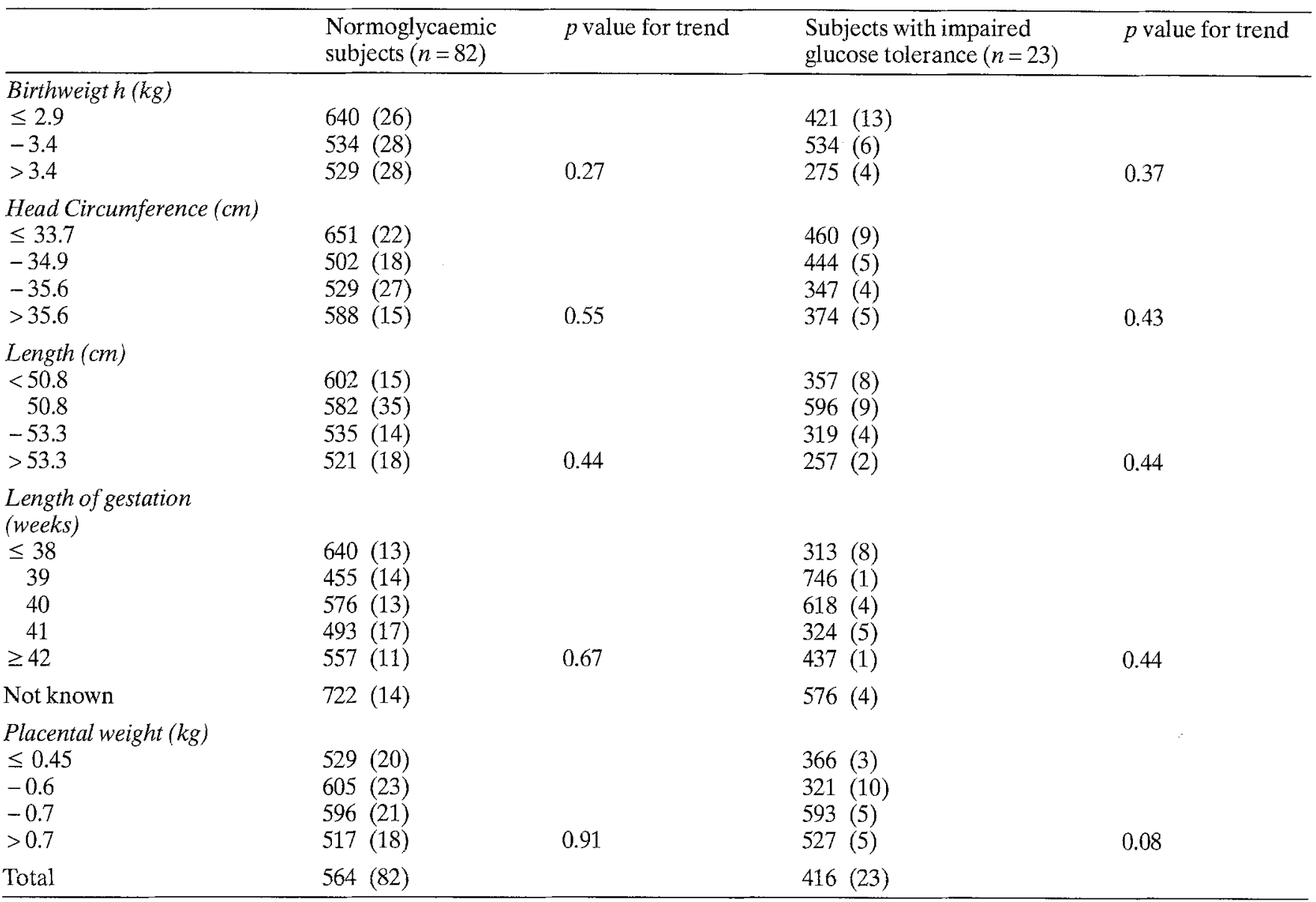

Numbers of subjects in parentheses;

Geometric standard deviation of 3-min plasma insulin $=1.86$

$10 \%$. Because the first phase insulin measurements had a skewed distribution, we transformed the distribution to normality using logarithms. The reported means in Tables 1 and 2 are therefore geometric means. Trend tests were carried out by means of one-way analysis of variance.

\section{Results}

The 82 normoglycaemic subjects were aged 47 to 55 years (mean 52 years) and had a mean body mass index of $25.9 \mathrm{~kg} / \mathrm{m}^{2}$, a mean fasting plasma glucose of $5.5 \mathrm{mmol} / 1$ and a mean fasting plasma insulin of $52 \mathrm{pmol} / \mathrm{l}$. The 23 subjects with glucose intolerance were of a similar age (range 47 to 55 years, mean 51 years). They had a higher body mass index, $26.7 \mathrm{~kg} / \mathrm{m}^{2}$, higher fasting plasma glucose, $6.0 \mathrm{mmol} / \mathrm{l}$, but similar fasting plasma insulin, $52 \mathrm{pmol} / \mathrm{l}$. The subjects with impaired glucose tolerance also had lower birthweight than the normoglycaemic subjects ( $2.9 \mathrm{vs} 3.1 \mathrm{~kg}, p=0.03)$ but the placental weights were similar, $0.6 \mathrm{~kg}$.

Table 1 shows mean insulin secretion 3 min after the administration of intravenous glucose in the normoglycaemic and glucose intolerant subjects according to measurements in the adults. The 82 normoglycaemic subjects had higher insulin secretion (mean 564 pmol/1) than the glucose intolerant subjects (mean $416 \mathrm{pmol} / \mathrm{l}$, $p=0.04)$. Men also had higher secretion $(601 \mathrm{pmol} / 1)$ than women ( $457 \mathrm{pmol} / \mathrm{l}, p=0.02)$. The differences between the normoglycaemic and the glucose intolerant subjects, and between men and women remained statistically significant after adjustment for current body mass index or gender or both. Insulin secretion tended to fall with increasing age although the trend was not significant $(p=0.44)$. Likewise there were nosignificant relationships between insulin secretion and body mass index $(p=0.38)$ or waist to hip ratio $(p=0.16)$. Insulin secretion correlated weakly with fasting insulin levels $(p=0.06)$. Insulin secretion was not associated with social class, either at birth or currently, or with smoking or alcohol consumption. We used multiple regression to analyse the simultaneous relations of glucose intolerance, sex, age, obesity and fasting insulin levels with insulin secretion. The effects of sex $(p=0.02)$, glucose intolerance $(p=0.01)$ and fasting insulin levels $(p=0.04)$ remained statistically significant but not age $(p=0.21)$ or body mass index $(p=0.83)$. 
Table 2 shows the mean 3-min insulin concentrations following the IVGTT according to measurements of the body size at birth. In this table we have used the same divisions of birth measurements as in previous analyses of data from Preston [2, 6]. Insulin secretion was not related to birthweight in either the normoglycaemic subjects or in the subjects with glucose intolerance ( $p=0.27$ and $p=0.37$ respectively). Neither was it related to head circumference ( $p=0.55$ and $p=0.43$ ), length ( $p=0.44$ in both groups), gestational age $(p=0.67$ and $p=0.44)$, or placental weight $(p=0.91$ and $p=0.08$ ). In addition, there was no correlation between insulin secretion and ponderal index or the ratio of the head circumference to length, indices which we have previously used to define babies who are thin at birth or disproportionately short $[2,6]$.

\section{Discussion}

We have studied the first phase insulin secretion in a group of men and women aged around 50 years whose body size at birth had been measured in detail. There was no relationship between insulin secretion and the measurements of prenatal growth in either the normoglycaemic or the glucose intolerant subjects.

Our measure of insulin secretion was the 3-min insulin concentration following the intravenous injection of insulin. We have shown that this measurement of first phase insulin release is highly reproducible [11]. In addition the first phase insulin response has been shown to correlate with both the pancreatic insulin content and beta-cell mass [14]. Consistent with the findings of other studies we found that insulin secretion was lower in the subjects with impaired glucose tolerance and was increased in more resistant individuals as indicated by a correlation between the 3-min insulin secretion and fasting insulin levels [15-17].

The failure to show a link between reduced fetal growth and insulin secretion is unlikely to have been due to the design of the study. The 105 subjects who took part in the study were born in hospital and still live in Preston, and are therefore unrepresentative of all men and women born in the town. However, as the analysis is based on internal comparisons it is unlikely that the selection of the sample would obscure correlations between fetal growth and insulin secretion. Nor was it likely to have been a consequence of the relatively small size of the study. Statistical power calculations indicate that a study of 105 subjects would be sufficient to have $84 \%$ power to detect a reduction of $20 \%$ in insulin secretion for each standard deviation $(0.52 \mathrm{~kg})$ drop in birthweight. Another possible explanation is that the pancreatic beta cells may hypertrophy in response to an increased demand for insulin with obesity and insulin resistance. The pattern of insulin secretion would then be determined by insulin resistance thereby obscuring associations with early growth patterns. This hypothesis is supported by our data which shows associations between insulin secretion andfasting insulin levels, reflecting insulin resistance, although these associations were relatively weak accounting for less than $5 \%$ of the variance in insulin secretion. The problem of insulin resistance obscuring relationships between early growth and insulin secretion could be overcome by studies of younger subjects and it is, therefore, of interest that in a study of young men aged 21 years we found that the 30 min insulin concentration during an OGTT was related to birthweight [18]. However, there is no apparent explanation for the very large differences in insulin secretion observed between individuals in the present study. They do not appear to be explained by differences in nutrition and growth in early life as measured by the anthropometric measurements to which we have access.

Our results are also at variance with previous studies showing that fetal or infant malnutrition in experimental animals or humans is associated with reduced insulin secretion [8-10]. However, these reports were based on severe protein or calorie undernutrition during pregnancy or in infancy or on the study of very low birthweight infants and are unlikely to have been typical of the conditions in Preston during 1935-1943. Nevertheless these studies do suggest that defects in insulin secretion could explain the links between reduced early growth and diabetes in adult life in human populations where malnutrition is very prevalent.

Together with the recently described association between insulin resistance and thinness in early life [6], our observations are evidence that in affluent western populations, the increased prevalence of diabetes in adults who were growth retarded during fetal life is mediated by increased insulin resistance rather than by abnormalities of insulin secretion.

Acknowledgements. Ms. L. Cox, Ms. M.Sheldon, Mr. R.Beck, Ms. F. Tulloch and Dr. N. Martensz performed the assays. The authors would like to thank Ms. J. Hart and Ms. M. Livesey for assistance with the fieldwork. The work was supported by the Medical Research Council, the British Diabetic Association and the British Heart Foundation. Ms. L. Bennett kindly typed the manuscript.

\section{References}

1. Hales CN, Barker DJP, Clark PMS et al. (1991) Fetal and infant growth and impaired glucose tolerance at age 64 years. BMJ 303: 1019-1022

2. Phipps K, Barker DJP, Hales CN, Fall CHD, Osmond C, Clark PMS (1993) Fetal growth and impaired glucose tolerance in men and women. Diabetologia 36: 225-228

3. Athens M, Valdez R, Stern M (1993) Effect of birthweight on future development of "Syndrome X" in adult life. Diabetes 42 [Suppl 1]: 61 a (Abstract)

4. McCance DR, Pettitt DJ, Hanson RL, Jacobsson LTH, Knowler WC, Bennett PH (1993) Low birthweight and type 2 diabetes in Pima Indians. Diabetologia 36 [Suppl 1]:A4 (Abstract) 
5. Barker DJP, Hales CN, Fall CHD, Osmond C, Phipps K, Clark PMS (1993) Type 2 (non-insulin-dependent) diabetes mellitus, hypertension and hyperlipidaemia (syndrome $\mathrm{X}$ ): relation to reduced fetal growth. Diabetologia 36: 62-67

6. Phillips DIW, Barker DJP, Hales CN, Hirst S, Osmond C (1994) Thinness at birth and insulin resistance in adult life. Diabetologia 37: 150-154

7. Van Assche FA, Aerts L (1979) The fetal endocrine pancreas. Contrib Gynecol Obstet 5: 44-57

8. Milner RDG (1971) Metabolic and hormonal responses to glucose and glucagon in patients with infantile malnutrition. Pediat Res 5: 33-39

9. Swenne I, Crace CJ, Milner RDG (1987) Persistent impairment of insulin secretory response to glucose in adult rats after limited period of protein-calorie malnutrition early in life. Diabetes 36: 454-458

10. Snoeck A, Remacle C, Reusens B, Hoet JJ (1990) Effect of a low protein diet during pregnancy on the fetal rat endocrine pancreas. Biol Neonate 5: 107-118

11. Rayman G, Clark P, Schneider AE, Hales CN (1990) The first phase insulin response to intravenous glucose is highly reproducible. Diabetologia 33: 631-634

12. Sobey WJ, Beer SF, Carrington CA et al. (1989) Sensitive and specific two-site immunoradiometric assay for the human insulin, and proinsulin, 65-66 split and 32-33 split proinsulins. Biochem J 260:535 -541

13. Alpha B, Cox L, Crowther N, Clark PMS, Hales CN (1992) Sensitive amplified immunoenzymometric assays (IEMA) for human insulin and intact proinsulin. Eur $\mathbf{J}$ Clin Chem Biochem 30: 27-32

14. McCulloch DK, Koerker DJ, Kahn SE et al. (1991) Correlations of in vivo beta cell function tests with beta cell mass and pancreatic insulin content in streptozocin-treated baboons. Diabetes 40: 673-679

15. Kosaka K, Hagura R, Kuzuya T (1977) Insulin responses in equivocal and definite diabetes, with special reference to subjects who had mild glucose intolerance but later developed definite diabetes. Diabetes 26: 944-952

16. Saad M, Knowler WC, Pettitt DJ, Nelson RG, Mott DM, Bennett PH (1988) The natural history of impaired glucose tolerance in the Pima Indians. New Engl J Med 319: 15001506

17. Bergman RN (1989) Toward physiological understanding of glucose intolerance. Diabetes 38: 1512-1527

18. Robinson SM, Walton RJ, Clark PMS, Barker DJP, Hales $\mathrm{CN}$, Osmond C (1992) The relation of fetal growth to plasma glucose in young men. Diabetologia 35: 444-446 